\title{
REVISTA CIENTÍFICA ODONTOLÓGICA
}

ISSN 2310-2594

\section{Revista Científica Odontológica}

Vol. $4 \mathrm{~N}^{\circ} 2$

Julio - Diciembre 2016

Lima-Perú

\section{DIRECTOR:}

o Mg. Luis Ernesto Arriola Guillén

\section{COMITÉ EDITORIAL:}

Mg. Guido Perona Miguel de Priego

Esp. Gustavo Huertas Mogollón

Dra. María Eugenia Guerrero Acebedo

Dra. Carmen Rosa García Rupaya

Mg. Sergio Alvarado Menacho

Mg. Gerardo Mendoza Azpur

Mg. Carol Cárdenas Flores

Mg. Romy Angeles Maslucán

Esp. Ana Isabel López Flores

\section{COMITÉ CONSULTIVO: \\ Mg. Rodolfo Valdivia Maibach \\ Mg. Elmer Salinas Prieto \\ Esp. Leslie Belmont Reátegui \\ Dr. Abraham Menéses López \\ Mg. Denisse Aguilar Gálvez \\ Esp. Gustavo Watanabe Oshiro \\ Dra. Marisol Castilla Camacho \\ Mg. Juan Manuel Farah Hayn \\ Mg. Patricia Vidal Manyari \\ Esp. Ana María Carlos Erazo \\ o Mg. Juan Price Rivera}

\section{COMITÉ CONSULTIVO INTERNACIONAL:}

\section{o Dr. Edgar Dávila}

Diplomado "American Board of Prosthodontics and the American Board of Oral Implantology", Tampa Advanced Dental Solutions (Estados Unidos)

o Dr. José Roberto Cortelli Periodoncia - Universidad de Taubaté (Brasil)

o Dr. Sigmar de Mello Rode Facultad de Odontología - Universidad de San José de los Campos / UNESP - Presidente de la Asociación Brasilera de Editores Científicos - ABEC (Brasil) - Editor Científico de la "Brazilian Oral Research"

- Dr. Rui V. Oppermann Periodoncia - Facultad de Odontología - Universidad de Río Grande del Sur (Brasil)

o Dr. Manuel De la Rosa Periodoncia e Implantología - Universidad de Monterrey (México)

o Dr. José Carlos Curvelo Prótesis - Universidad Paulista de Odontología (Brasil) - Máster en Implantología, SL Mandic

Dr. Valter Castro Alves Máster en Implantología - Universidad Unicastelo (Brasil)

o Dr. Claudio Mendes Pannuti

Máster en Implantología - Universidad Unicastelo (Brasil) 